\title{
Effective a posteriori co-phasing of interferometric fringe data
}

\author{
Antony Schutz ${ }^{\mathrm{a}}$, Éric Thiébaut ${ }^{\mathrm{b}}$, Ferréol Soulez ${ }^{\mathrm{b}, \mathrm{c}}$, Michel Tallon ${ }^{\mathrm{b}}$, Gilles Duvert ${ }^{\mathrm{d}}$, and Isabelle \\ Tallon-Bosc ${ }^{\mathrm{b}}$ \\ ${ }^{a}$ Lab. J.-L. Lagrange, Université de Nice Sophia Antipolis, CNRS, Observatoire de la Côte d'Azur, Parc Valrose, \\ F-06108 Nice cedex 02, France \\ ${ }^{\mathrm{b}}$ Univ. Lyon, Univ. Lyon 1, ENS de Lyon, CNRS, Centre de Recherche Astrophysique de Lyon UMR5574, F-69230, \\ Saint-Genis-Laval, France \\ ${ }^{\mathrm{c}}$ Biomedical Imaging Group, École Polytechnique Fédérale de Lausanne (EPFL), CH-1015 Lausanne, Switzerland. \\ ${ }^{\mathrm{d}}$ Institut de Planétologie et d'Astrophysique de Grenoble, France
}

\begin{abstract}
We have recently shown that a posteriori co-phasing of multi-spectral interferograms was possible. ${ }^{1}$ In this contribution, we extend our approach so that it can be applied to actual data as provided by AMBER ${ }^{2}$ or MATISSE instruments. The main advantage of the proposed post-processing technique is that it requires no modifications of the instruments and yields interferometric observables with higher SNR and much fewer unknowns (in particular for the Fourier phase) than conventional measurements. In order to perform the co-phasing of a complete sequence of interferograms, we jointly estimate a global phase template and the frame dependent optical path errors due to the turbulence. We show that this strategy is effective for very low SNR data. We assess the effectiveness of our method on simulated and actual AMBER data. We also compare the lowest SNR that can be achieved to the theoretical bounds and estimate the gain in sensitivity compared to usual interferometric data.
\end{abstract}

Keywords: optical interferometry, cophasing, phase delay tracking, dispersed fringes, data processing.

\section{METHOD}

To overcome turbulence effects and yet reach a reasonable signal to noise ratio (SNR), interferometric observables require to integrate information over many short exposure frames computed from the so-called coherent fluxes. ${ }^{3,4}$ Our objective is to compensate for the variable phase changes during a given sequence so that it is possible to perform a direct integration of the coherent fluxes over many short exposures, before the computation of the long exposure chromatic complex visibilities.

\subsection{Maximum Likelihood Criterion}

Let $c_{\ell, m} \in \mathbb{C}$ be the coherent flux measured in $\ell$-th spectral channel and $m$-th frame; it is related to the complex visibility $c_{\ell}^{\mathrm{obj}} \in \mathbb{C}$ of the observed object at the wavelength $\lambda_{\ell}$ of the spectral channel by: $:^{3,4}$

$$
c_{\ell, m}=c_{\ell, m}^{\mathrm{atm}} c_{\ell}^{\mathrm{inst}} c_{\ell}^{\mathrm{obj}}+n_{\ell, m},
$$

where $c_{\ell}^{\text {inst }} \in \mathbb{C}$ is a static instrumental complex visibility, $c_{\ell, m}^{\text {atm }} \in \mathbb{C}$ is a variable complex factor mainly due to atmospheric effects and $n_{\ell, m}$ accounts for the noise. Our objective is to provide an estimator closely related to $c_{\ell}^{\text {stat }} \stackrel{\text { def }}{=} c_{\ell}^{\text {inst }} c_{\ell}^{\text {obj }}$, the static part of the coherent flux. Getting rid of the $c_{\ell}^{\text {inst }}$ factor is a matter of calibrating this term either by means of internal calibration sources or by observing a calibrator whose complex visibility is known.

Since we are interested in compensating for variable phase shifts, we rewrite the direct model in Eq. (1) as:

$$
c_{\ell, m}=\rho_{\ell, m} \mathrm{e}^{\mathrm{i}\left(\varphi_{\ell}+\psi_{\ell, m}\right)}+n_{\ell, m},
$$

E-mail: eric.thiebaut@univ-lyon1.fr

Optical and Infrared Interferometry and Imaging V, edited by Fabien Malbet, 
where

$$
\rho_{\ell, m}=\left|c_{\ell, m}^{\mathrm{atm}} c_{\ell}^{\mathrm{inst}} c_{\ell}^{\mathrm{obj}}\right|
$$

is a variable amplitude factor, $\varphi_{\ell}$ is the phase of $c_{\ell}^{\text {stat }}=c_{\ell}^{\text {inst }} c_{\ell}^{\text {obj }}$ and $\psi_{\ell, m}=\operatorname{angle}\left(c_{\ell, m}^{\text {atm }}\right)$ is the phase of $c_{\ell, m}^{\text {atm }}$.

We assume that the measured coherent fluxes are Gaussian independent, that the real and imaginary parts of a given coherent flux are also independent and have the same variance, in agreement with Goodman model. ${ }^{5}$ We also assume that the variable phases $\psi_{\ell, m}(\forall \ell, \forall m)$ depend on unknown parameters $\theta$. Under our assumptions, the co-log-likelihood of the data is given by: ${ }^{1}$

$$
\begin{aligned}
-\log \operatorname{Pr}(c \mid \varphi, \theta, \rho) & =\frac{1}{2} \sum_{\ell, m} w_{\ell, m}\left|c_{\ell, m}-\rho_{\ell, m} \mathrm{e}^{\mathrm{i}\left(\varphi_{\ell}+\psi_{\ell, m}(\theta)\right)}\right|^{2}+\kappa \\
& =\frac{1}{2} \sum_{\ell, m} w_{\ell, m}\left(\left|c_{\ell, m}\right|^{2}+\rho_{\ell, m}^{2}\right)-\sum_{\ell, m} w_{\ell, m} \rho_{\ell, m}\left|c_{\ell, m}\right| \cos \left(\varphi_{\ell}+\psi_{\ell, m}(\theta)-\phi_{\ell, m}\right)+\kappa,
\end{aligned}
$$

with $w_{\ell, m} \geq 0$ a statistical weight equal to the reciprocal of the variance of the real and imaginary parts of $c_{\ell, m}$, $\phi_{\ell, m}=\operatorname{angle}\left(c_{\ell, m}\right)$ the phase of $c_{\ell, m}$ and $\kappa$ a constant which is irrelevant as it does not depend on the sought parameters.

\subsection{Fitting all the unknowns}

Following a maximum likelihood approach and if the amplitudes $\rho_{\ell, m}$ are unknown, we would estimate the best phases $\varphi_{\ell}$ as:

$$
\widehat{\varphi} \in \underset{\varphi \in(-\pi,+\pi]^{L}}{\arg \min }\left\{\min _{\theta, \rho \geq 0} \sum_{\ell, m} w_{\ell, m}\left|c_{\ell, m}-\rho_{\ell, m} \mathrm{e}^{\mathrm{i}\left(\varphi_{\ell}+\psi_{\ell, m}(\theta)\right)}\right|^{2}\right\},
$$

where $L$ is the number of spectral channels and $\varphi=\left\{\varphi_{1}, \varphi_{2}, \ldots, \varphi_{L}\right\}$ are the $L$ sought phases defined in the semi-open range $(-\pi,+\pi]$. We use the notation " $\widehat{\varphi} \in \ldots$. because the solution may not be unique (apart from the modulo $2 \pi)$. The minimization with respect to every $\rho_{\ell, m}$ is separable and has a closed form solution ${ }^{1}$ (provided $w_{\ell, m}>0$ ):

$$
\min _{\rho_{\ell, m} \geq 0} w_{\ell, m}\left|c_{\ell, m}-\rho_{\ell, m} \mathrm{e}^{\mathrm{i}\left(\varphi_{\ell}+\psi_{\ell, m}(\theta)\right)}\right|^{2}=w_{\ell, m}\left|c_{\ell, m}\right|^{2}\left[1-\left(\cos \left(\varphi_{\ell}+\psi_{\ell, m}(\theta)-\phi_{\ell, m}\right)\right)_{+}^{2}\right],
$$

where $(t)_{+} \stackrel{\text { def }}{=} \max \{t, 0\}$. Inserting this solution in Eq. (6) yields:

$$
\widehat{\varphi} \in \underset{\varphi \in(-\pi,+\pi]^{L}}{\arg \min }\left\{\min _{\theta} \sum_{\ell, m} w_{\ell, m}\left|c_{\ell, m}\right|^{2}\left[1-\left(\cos \left(\varphi_{\ell}+\psi_{\ell, m}(\theta)-\phi_{\ell, m}\right)\right)_{+}^{2}\right]\right\} .
$$

We have already shown ${ }^{1}$ that fitting the amplitudes $\rho_{\ell, m}$ does not achieve the best performances for the cophasing of frames. This can be understood from Eq. (7) that shows a truncated criterion. Indeed, because of the $(t)_{+}$operator, the penalization does not change anymore as soon as the cosine is negative and cannot pull down to the minimum.

\subsection{Fitting the phase parameters only}

A better approach is to fit only the phase parameters, assuming that all the $\rho_{\ell, m}$ are known, the maximum likelihood phase parameters are obtained by solving:

$$
\min _{\varphi, \theta}\left\{f(\varphi, \theta)=\sum_{\ell, m} w_{\ell, m}\left|c_{\ell, m}-\rho_{\ell, m} \mathrm{e}^{\mathrm{i}\left(\varphi_{\ell}+\psi_{\ell, m}(\theta)\right)}\right|^{2}\right\} .
$$

By setting $u_{\ell, m}=c_{\ell, m}, v_{\ell, m}=\rho_{\ell, m} \mathrm{e}^{\mathrm{i} \psi_{\ell, m}(\theta)}$ and $\chi=\varphi$, minimizing $f(\varphi, \theta)$ with respect to $\varphi$ corresponds to solving Problem (24) in Appendix A. The penalty $f(\varphi, \theta)$ in Eq.(8) can thus be expressed as:

$$
f(\varphi, \theta)=\sum_{\ell, m} w_{\ell, m}\left(\left|c_{\ell, m}\right|^{2}+\rho_{\ell, m}^{2}\right)-2 g(\varphi, \theta),
$$


with:

$$
g(\varphi, \theta)=\sum_{\ell, m} \eta_{\ell, m} \cos \left(\varphi_{\ell}+\psi_{\ell, m}(\theta)-\phi_{\ell, m}\right)
$$

where:

$$
(\forall \ell, m) \quad \eta_{\ell, m}=w_{\ell, m} \rho_{\ell, m}\left|c_{\ell, m}\right|,
$$

play the role of weights. Then Problem (8) is equivalent to:

$$
\min _{\varphi, \theta} f(\varphi, \theta) \Longleftrightarrow \max _{\varphi, \theta} g(\varphi, \theta) .
$$

Assuming known the variable phase parameters $\theta$ and from Eq. (27), the best static phases are given by:

$$
(\forall \ell) \quad \widehat{\varphi}_{\ell}(\theta)=\operatorname{angle}\left(\sum_{m} \eta_{\ell, m} \cos \left(\phi_{\ell, m}-\psi_{\ell, m}(\theta)\right)+\mathrm{i} \sum_{m} \eta_{\ell, m} \sin \left(\phi_{\ell, m}-\psi_{\ell, m}(\theta)\right)\right),
$$

In Appendix A, with $u_{\ell, m}=c_{\ell, m}$ and $v_{\ell, m}=\rho_{\ell, m} \mathrm{e}^{\mathrm{i} \psi_{\ell, m}(\theta)}$ as before, the angles $\xi$ become $\xi_{\ell, m}=\operatorname{angle}\left(u_{\ell, m} v_{\ell, m}^{\star}\right)=$ $\phi_{\ell, m}-\psi_{\ell, m}(\forall \ell, \forall m)$. Then, from Eq. (30) and after elementary simplifications, the best variable phase shift parameters $\theta$ can be obtained by solving:

$$
\widehat{\theta}=\underset{\theta}{\arg \max }\left\{g(\theta)=\sum_{\ell} \sqrt{\sum_{m, m^{\prime}} \eta_{\ell, m} \eta_{\ell, m^{\prime}} \cos \left(\Delta \phi_{\ell, m, m^{\prime}}-\Delta \psi_{\ell, m, m^{\prime}}(\theta)\right)}\right\},
$$

where:

$$
\begin{aligned}
\Delta \phi_{\ell, m, m^{\prime}} & =\phi_{\ell, m^{\prime}}-\phi_{\ell, m}, \\
\Delta \psi_{\ell, m, m^{\prime}}(\theta) & =\psi_{\ell, m^{\prime}}(\theta)-\psi_{\ell, m}(\theta) .
\end{aligned}
$$

We note that $\Delta \phi_{\ell, m, m^{\prime}}$ is also the phase of the cross-product $c_{\ell, m}^{\star} c_{\ell, m^{\prime}}$ of the coherent fluxes $c_{\ell, m}$ and $c_{\ell, m^{\prime}}$. The criterion $f(\theta)$ given in Eq. (14) is thus clearly insensitive to the actual phase of the object complex visibility which is an important property for the co-phasing of the data.

\subsection{Model of the phase variations}

Accounting for an achromatic variable phase shift $\psi_{0}(t)$ and a chromatic and variable optical path delay $\delta(\lambda, t)$, the resulting phase shift is given by:

$$
\psi(\lambda, t)=\psi_{0}(t)+\frac{2 \pi \delta(\lambda, t)}{\lambda} .
$$

A Taylor series in the form:

$$
\delta(\lambda, t)=\delta\left(\lambda_{0}, t\right)+\left.\left(\lambda-\lambda_{0}\right) \frac{\partial \delta(\lambda, t)}{\partial \lambda}\right|_{\lambda=\lambda_{0}}+\ldots
$$

can be used to approximate the chromatic behavior of the optical path delay and to yield the following model:

$$
\psi(\lambda, t) \approx \beta(t)+\alpha(t) / \lambda,
$$

where $\alpha(t)$ and $\beta(t)$ are some time dependent functions. For the $\ell$-th spectral channel and $m$-th frame, this leads us to assume the following model of the variable phase:

$$
\psi_{\ell, m}=\beta_{m}+\alpha_{m} / \lambda_{\ell},
$$

with $\alpha_{m}=\alpha\left(t_{m}\right)$ and $\beta_{m}=\beta\left(t_{m}\right)$. The variable phase parameters are thus: $\theta=\left\{\alpha_{m}, \beta_{m} \mid m=1, \ldots, M\right\}$ with $M$ the number of short exposure frames.

We consider in what follows means to determine these parameters. Maximizing $f(\theta)$ defined in Eq. (14) is a very difficult non-concave problem which can only be solved by means of global optimization. We propose to tackle this problem by first co-phasing two chosen frames and then iteratively add frames and perform the co-phasing individually (one frame at a time). 


\section{Cost function for different phase shift models}

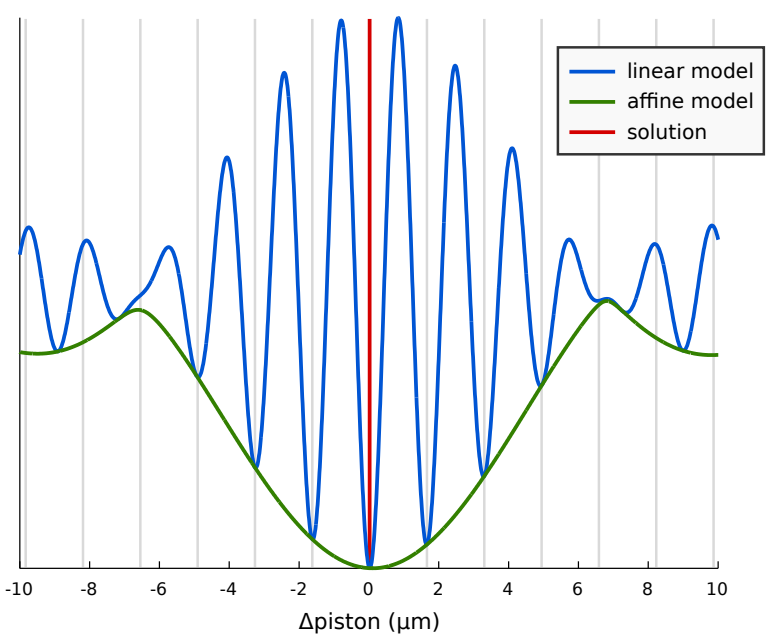

Figure 1. Criterion to minimize for the co-phasing of two frames as a function of $\Delta \alpha$. The blue curve corresponds to $-g(\Delta \alpha, \Delta \beta=0)$ while the red curve corresponds to $-g(\Delta \alpha)$. The curves are from two frames drawn from AMBER data as described in the text.

\section{Cost function for different SNR}

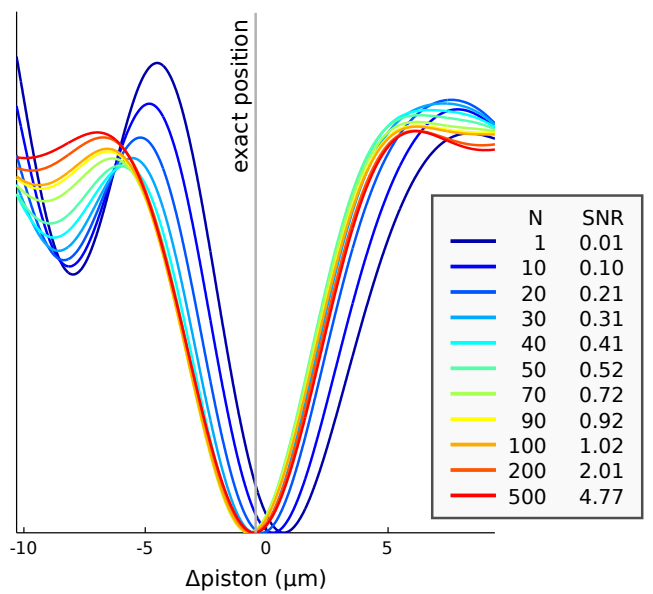

Figure 2. Criterion to minimize for different SNR. The true solution is indicated by the vertical gray line.

\subsection{Co-phasing of two frames}

Using the variable phase model in Eq. (18), we can write:

$$
\Delta \psi_{\ell, m, m^{\prime}}=\psi_{\ell, m^{\prime}}-\psi_{\ell, m}=\Delta \beta_{m, m^{\prime}}+\Delta \alpha_{m, m^{\prime}} / \lambda_{\ell},
$$

with $\Delta \alpha_{m, m^{\prime}}=\alpha_{m^{\prime}}-\alpha_{m}$ and $\Delta \beta_{m, m^{\prime}}=\beta_{m^{\prime}}-\beta_{m}$ which characterize the differential phase shift between the frames $m$ and $m^{\prime}$. However, maximizing $f(\theta)$ given in Eq. (14) with respect to $\Delta \alpha_{m, m^{\prime}}$ and $\Delta \beta_{m, m^{\prime}}$ turns out to be a $2 \mathrm{D}$ global optimization problem which cannot be further reduced to a simpler problem. We therefore propose to minimize the co-log-likelihood, in Eq. (5), of the two frames, $m$ and $m^{\prime}$, but replacing the (unknown) phase shift by the phase in the other frame with its variable phase shift removed. This amounts to maximizing:

$$
g(\Delta \alpha, \Delta \beta)=\sum_{\ell}\left(\eta_{\ell, m}+\eta_{\ell, m^{\prime}}\right) \cos \left(\Delta \phi_{\ell, m, m^{\prime}}-\Delta \beta-\Delta \alpha / \lambda_{\ell}\right),
$$

with respect to $\Delta \alpha$ and $\Delta \beta$ and to estimate the differential phase shift parameters as $\Delta \alpha_{m, m^{\prime}}=\widehat{\Delta \alpha}$ and $\Delta \beta_{m, m^{\prime}}=\widehat{\Delta \beta}$ where:

$$
\widehat{\Delta \alpha}, \widehat{\Delta \beta}=\underset{\Delta \alpha, \Delta \beta}{\arg \max } g(\Delta \alpha, \Delta \beta) .
$$

As shown in Appendix $\mathrm{A}$, the maximization in $\Delta \beta$ has a closed form solution:

$$
\widehat{\Delta \beta}(\Delta \alpha)=\underset{\Delta \beta}{\arg \max } g(\Delta \alpha, \Delta \beta)=\operatorname{angle}(x(\Delta \alpha)+\mathrm{i} y(\Delta \alpha)) .
$$

with:

$$
\begin{aligned}
& x(\Delta \alpha)=\sum_{\ell}\left(\eta_{\ell, m}+\eta_{\ell, m^{\prime}}\right) \cos \left(\Delta \phi_{\ell, m, m^{\prime}}-\Delta \alpha / \lambda_{\ell}\right), \\
& y(\Delta \alpha)=\sum_{\ell}\left(\eta_{\ell, m}+\eta_{\ell, m^{\prime}}\right) \sin \left(\Delta \phi_{\ell, m, m^{\prime}}-\Delta \alpha / \lambda_{\ell}\right) .
\end{aligned}
$$

Replacing this solution in $g(\Delta \alpha, \Delta \beta)$ yields a criterion which only depends on $\Delta \beta$ :

$$
g(\Delta \alpha)=\sqrt{x(\Delta \alpha)^{2}+y(\Delta \alpha)^{2}} .
$$


Fringe SNR

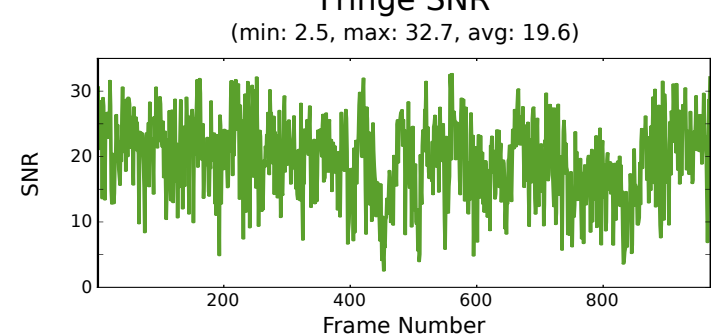

Coherent Flux Amplitude

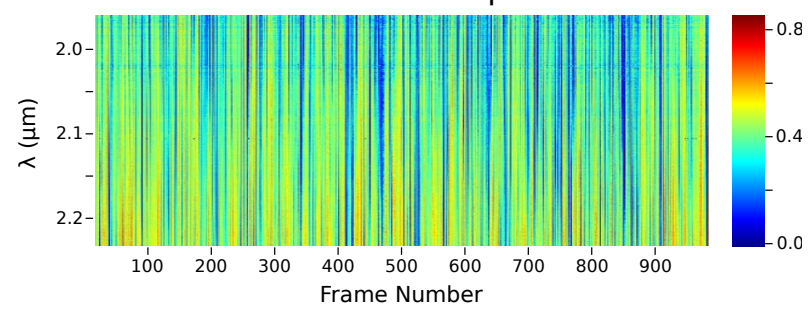

Coherent Flux Phase

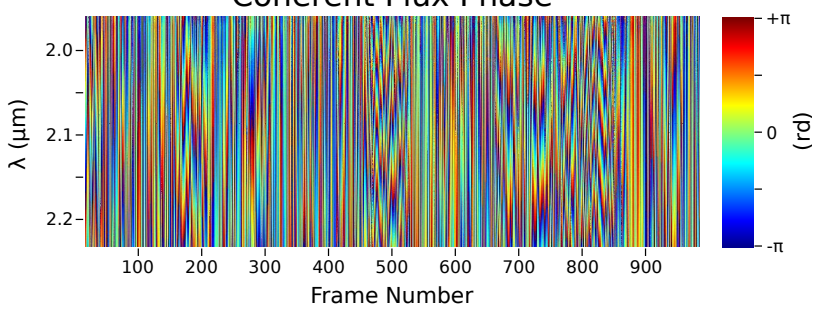

Cophased with the piston computed by AMDLIB

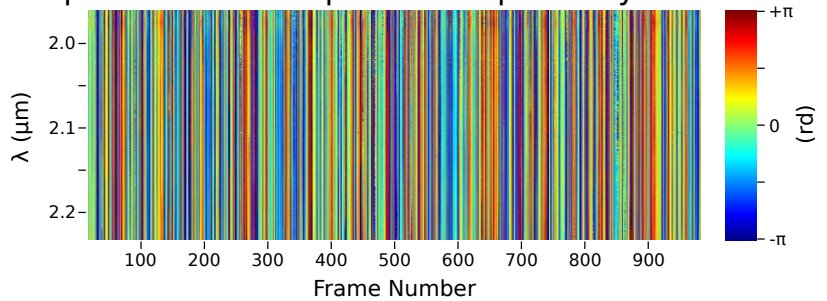

Cophased by the proposed method

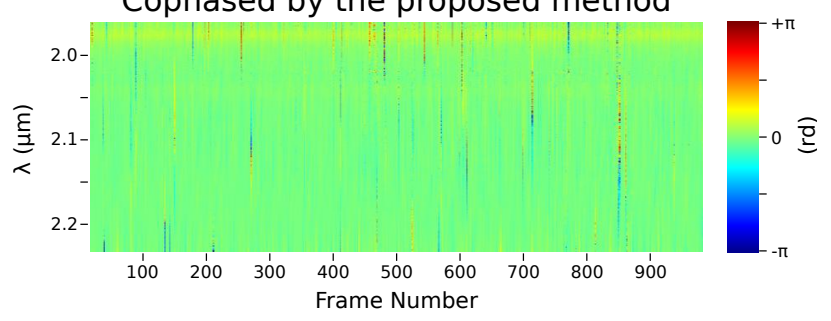

Restored Phase

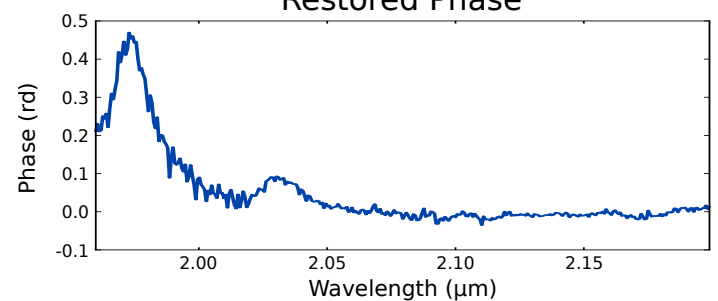

Figure 3. Application to all frames of a sequence of AMBER frames. From top to bottom and left to right: the SNR of the 980 frames; the amplitudes of the coherent flux in each frame; the phases of the coherent flux in each frame; the phases of the coherent flux after accounting for the piston computed by AMDLIB; the phases of the coherent flux after applying the proposed method; the resulting static phase for all the sequence.

It is then sufficient to maximize $g(\Delta \alpha)$ using the global 1D optimization method described in. ${ }^{1}$ Figure 1 shows the behavior of $-g(\Delta \alpha)$ and, for comparison, $-g(\Delta \alpha, \Delta \beta=0$ ) (thus the optimal corresponds to the global minimum in this figure). Clearly, assuming $\Delta \beta=0$ yields a criterion with many more local extrema.

In order to have the best robustness, it is preferable to apply the two-frame co-phasing method to the pair of frames which have the highest SNR. Given the values of $\widehat{\Delta \alpha}$ and $\widehat{\Delta \beta}$, an initial estimate of the static phase can be obtained by applying formula (13). There is however a degeneracy here as we only have the requirements that $\widehat{\Delta \alpha}=\alpha_{m^{\prime}}-\alpha_{m}$ and $\widehat{\Delta \beta}=\beta_{m^{\prime}}-\beta_{m}$, for instance we take:

$$
\begin{array}{ll}
\alpha_{m^{\prime}}=+\widehat{\Delta \alpha} / 2, & \alpha_{m}=-\widehat{\Delta \alpha} / 2, \\
\beta_{m^{\prime}}=+\widehat{\Delta \beta} / 2, & \beta_{m}=-\widehat{\Delta \beta} / 2 .
\end{array}
$$

In the next section, we explain how we perform the co-phasing of the full sequence.

\subsection{Co-phasing of a sequence of frames}

In order to co-phase many frames, we cannot directly solve Problem Eq. (8). So we propose to proceed iteratively by the following alternating method:

0. Initialization. Choose an initial estimate of the static phase $\widehat{\varphi}^{(0)}$ (for instance by co-phasing the 2 frames which have the best SNR as explained in Section 1.5). Then for $k=1,2, \ldots$ and until convergence, repeat the two following steps. 

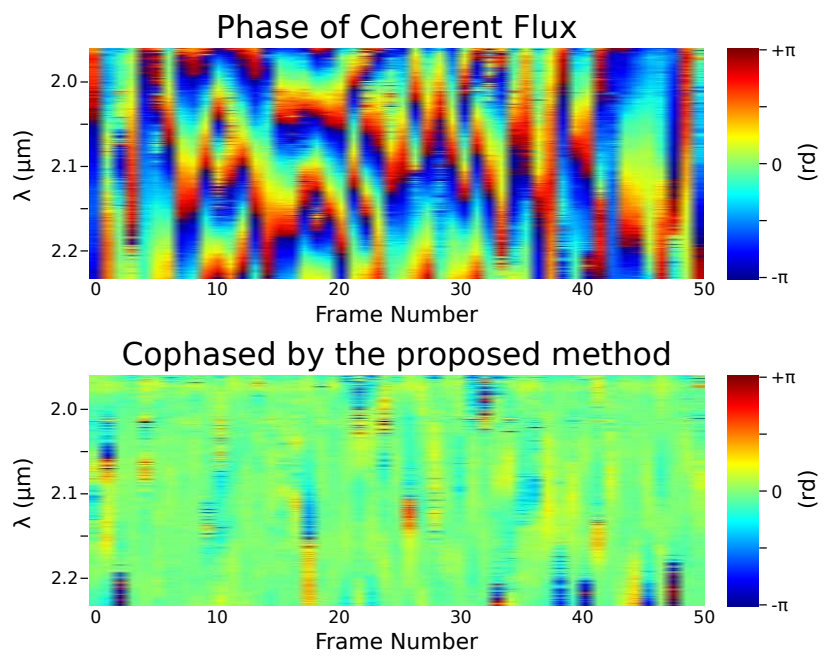
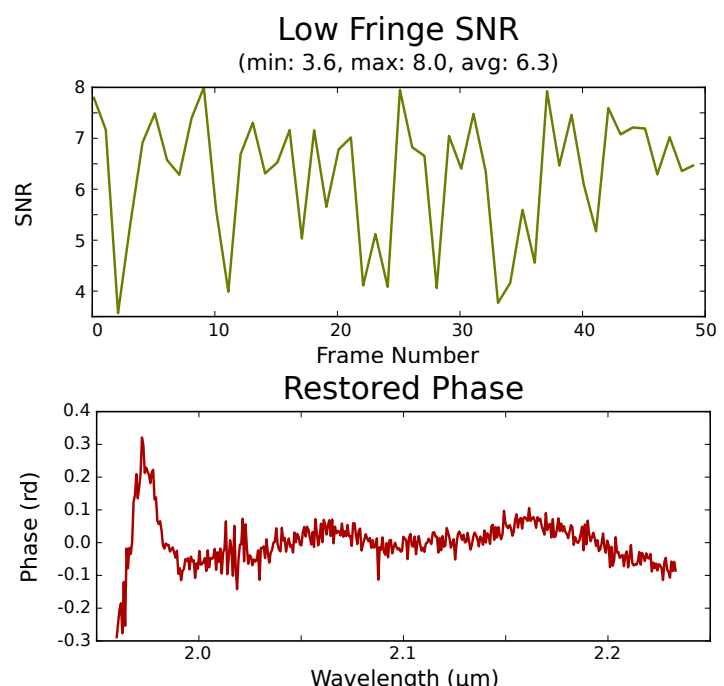

Figure 4. Application to 50 (out of 980 of a sequence of AMBER data) frames with a low SNR. From top to bottom and left to right: the phases of the coherent flux in each frame; the phases of the coherent flux after applying the proposed method; the SNR of each frames; the resulting static phase for all the sequence.

1. Co-phasing of frames. At iteration $k$, we assume the static phases given by $\widehat{\varphi}^{(k-1)}$ and, according to Eq. (8), we solve:

$$
\widehat{\theta}^{(k)}=\underset{\theta}{\arg \max } f\left(\widehat{\varphi}^{(k-1)}, \theta\right),
$$

to achieve the co-phasing of all frames. With the assumed variable phase model, in Eq. (18), the above problem is separable in independent problems with respect to the frame number $m$ and co-phasing the set of frames assuming known the static phase $\widehat{\varphi}^{(k-1)}$ amounts to solving:

$$
\max _{\alpha, \beta} \sum_{\ell, m} \eta_{\ell, m} \cos \left(\widehat{\varphi}_{\ell}^{(k-1)}+\alpha_{m} / \lambda_{\ell}+\beta_{m}-\phi_{\ell, m}\right),
$$

which is separable in $m$. According to Appendix A:

$$
\begin{aligned}
\widehat{\beta}_{m}\left(\alpha_{m}\right) & =\underset{\beta_{m}}{\arg \max } \sum_{\ell} \eta_{\ell, m} \cos \left(\widehat{\varphi}_{\ell}^{(k-1)}+\alpha_{m} / \lambda_{\ell}+\beta_{m}-\phi_{\ell, m}\right) \\
& =\operatorname{angle}(\underbrace{\sum_{\ell} \eta_{\ell, m} \cos \left(\phi_{\ell, m}-\widehat{\varphi}_{\ell}^{(k-1)}-\alpha_{m} / \lambda_{\ell}\right)}_{x_{m}^{(k)}\left(\alpha_{m}\right)}+\mathrm{i} \underbrace{\sum_{\ell} \eta_{\ell, m} \sin \left(\phi_{\ell, m}-\widehat{\varphi}_{\ell}^{(k-1)}-\alpha_{m} / \lambda_{\ell}\right)}_{y_{m}^{(k)}\left(\alpha_{m}\right)}),
\end{aligned}
$$

and the optimal $\alpha_{m}$ can then be obtained by solving separable 1D global optimization problems:

$$
(\forall m) \quad \widehat{\alpha}_{m}^{(k)}=\underset{\alpha_{m}}{\arg \max } \sqrt{\left[x_{m}^{(k)}\left(\alpha_{m}\right)\right]^{2}+\left[y_{m}^{(k)}\left(\alpha_{m}\right)\right]^{2}} .
$$

Then $\widehat{\theta}^{(k)}=\left\{\widehat{\alpha}_{m}^{(k)}, \widehat{\beta}^{(k)} \mid m=1,2, \ldots\right\}$ with $\widehat{\beta}_{m}^{(k)}=\widehat{\beta}_{m}\left(\widehat{\alpha}_{m}^{(k)}\right)$ is the solution of Problem (21).

2. Estimation of the static phase. We use Eq. (13) to compute a new estimate of the static phase:

$$
\widehat{\varphi}^{(k)}=\operatorname{angle}\left(\sum_{m} \eta_{\ell, m} \cos \left(\phi_{\ell, m}-\psi_{\ell, m}\left(\widehat{\theta}^{(k)}\right)\right)+\mathrm{i} \sum_{m} \eta_{\ell, m} \sin \left(\phi_{\ell, m}-\psi_{\ell, m}\left(\widehat{\theta}^{(k)}\right)\right)\right) .
$$

In practice, we start with the two frames with the highest SNR and then add frames by decreasing SNR. For difficult cases, it may be more robust to iterate and refine $\widehat{\theta}$ before adding new frames. The two last panels of Fig. 3 show the effectiveness of the proposed method on a set of 980 AMBER frames. 

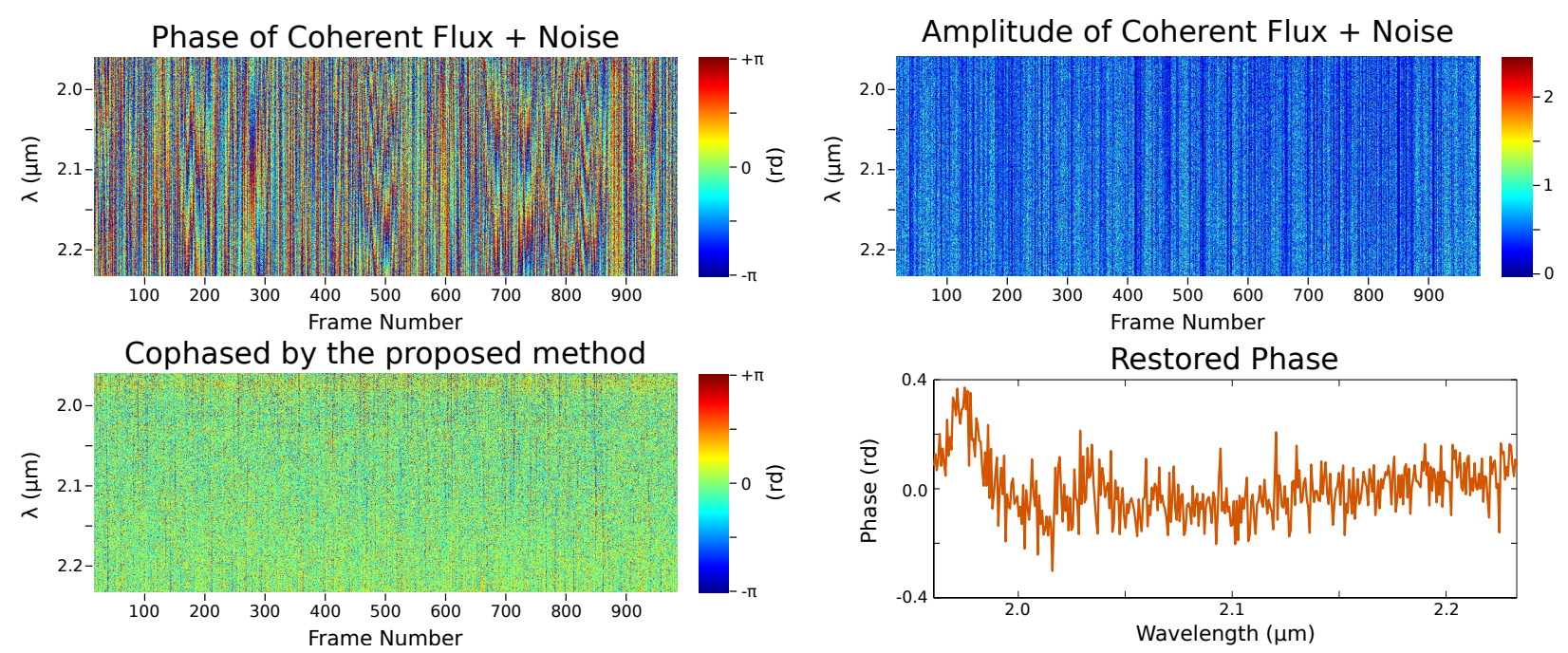

Figure 5. Application to 980 frames with a SNR $\simeq 1$. Artificial noise has been added to actual AMBER data so as to obtain very low SNR coherent fluxes. From top to bottom and left to right: the phases of the coherent flux in each frame; the phases of the coherent flux after applying the proposed method; the amplitudes of the coherent flux in each frame; the resulting static phase for all the sequence.

\section{RESULTS}

We applied the proposed method to coherent fluxes measured by AMBER. The data set is a sequence of 980 short exposure frames with 364 spectral channels in the $\mathrm{K}$ band (medium resolution of AMBER). The average fringe signal to noise ratio (SNR) is about 20 (see upper left panel in Fig. 3). The amplitudes and phases of the measured coherent fluxes are displayed by the middle and bottom panels of the leftmost column of Fig. 3. The phases appear very different from one frame to another although there is some correlations between successive frames. The upper right panel in Fig. 3 shows the phases of the coherent fluxes co-phased using the piston computed by AMBER pipeline ${ }^{6}$ (AMDLIB). This procedure is inappropriate as it destroys the aforementioned correlations. By comparison, the phases of the coherent fluxes co-phased by our method (shown in the middle panel of the rightmost column of Fig. 3) are much more stable across the temporal (horizontal) axis. In particular a phase peak can be seen on individual frames around $1.98 \mu \mathrm{m}$. The static phase $\widehat{\varphi}$ estimated by the proposed alternating algorithm is shown in the lower right panel of Fig. 3. After calibration to remove any static instrumental effects, this phase corresponds to that of the complex visibility of the observed object up to an additive chromatic correction given by:

$$
\psi(\lambda)=\beta+\alpha / \lambda,
$$

which follows from Eq. (18). The 2 parameters $\alpha$ and $\beta$ are not provided by the proposed method and have to be estimated by other means. The phase of the differential visibilities ${ }^{7}$ which can be provided by AmBER pipeline also have such degeneracies.

In order to check whether the proposed method can achieve an effective co-phasing with data of reduced quality, we processed a subset of only 50 frames of much lower SNR (6.3 on average) extracted from the same data set. Figure 4 shows that, in spite of the severely reduced quality of this subset of data, our method was able to estimate a static phase (shown in the lower right panel of Fig.4) which is noisier but which, apart from a slope due to the degeneracy, has the same shape as the phase estimated from the full data set.

The ability to perform co-phasing of the frames is certainly related to the fringe SNR. To study the effects of the SNR on the proposed method, we artificially add some independent noise to the measured coherent fluxes to reduce their SNR to a specific lower value. Figure 2 shows how the estimated parameter $\Delta \alpha_{m, m^{\prime}}$ is affected by the SNR: the lower the fringe SNR the worse the estimate. However the co-phasing of two frames seems still possible with SNR $\lesssim 0.1$ per spectral channel which is much lower than unity. This is not surprising considering that the information from many (here 164) spectral channels is jointly used to perform the co-phasing. Figure 5 shows the result of applying our method to the sequence of 980 frames with added noise so that their SNR is 
about 1 per spectral channel. The restored phase clearly shares distinctive features with the one obtained with a data set of much higher quality.

\section{SUMMARY AND PERSPECTIVES}

Following our preliminary work, ${ }^{1}$ we derive a method to estimate the time-varying phase shifts that affect coherent fluxes measured by an interferometric instrument like AMBER ${ }^{2}$ or MATisse. The proposed method exploits a chromatic model of the variable phase shifts to estimate and then compensate these shifts. In principle, cophasing by fitting the model of the phase shifts is optimal, in a maximum likelihood sense, but requires to solve a highly involved global optimization problem with many parameters. We propose to use an alternating method where the order of the various estimations are carefully selected to effectively solve the complex problem by solving more simple independent univariate global optimization problems. Applied on actual AMBER data, our method seems very effective even for data of very low quality.

In a very near future, we will consider estimating not only the phases but also the amplitudes by extending the proposed algorithm. Implementing the method in AMBER or MATISSE pipelines would be quite straightforward and could yield interferometric observables of improved quality. It would also relax the requirements for the fringe tracker. A more extensive study on simulated and actual data is also needed to characterize the performances and limitations of the method.

\section{ACKNOWLEDGMENTS}

This work was granted access to the HPC and visualization resources of "Centre de Calcul Interactif" hosted by "Université Nice Sophia Antipolis".

The research leading to these results has received funding from the European Community's Seventh Framework Programme (FP7/2013-2016) under grant agreement number 312430 (OPTICON).

The research leading to these results has received support from the MORIN project funded by the "Programme Avenir Lyon-Saint Étienne" (PALSE) of the University of Lyon in the framework of the program "Investissement d'Avenir" (ANR-11-IDEX-0007).

\section{APPENDIX A. TYPICAL CO-PHASING PROBLEM}

We consider the following problem:

$$
\min _{\chi \in(-\pi,+\pi]^{N}}\left\{f(\chi)=\sum_{j, k} w_{j, k}\left|u_{j, k}-v_{j, k} \mathrm{e}^{\mathrm{i} \chi_{j}}\right|^{2}\right\}
$$

where $\chi=\left\{\chi_{1}, \chi_{2}, \ldots, \chi_{N}\right\}$ are $N$ unknown phases defined in the semi-open range $(-\pi,+\pi], w_{j, k} \geq 0$ are nonnegative weights while $u_{j, k} \in \mathbb{C}$ and $v_{j, k} \in \mathbb{C}$ are given complexes. Of course, the problem above is separable in $j$, but this specific form is typical to several problems considered in this paper. Expanding the squares, the criterion to minimize can be rewritten as:

$$
f(\chi)=\sum_{j, k} w_{j, k}\left(\left|u_{j, k}\right|^{2}+\left|v_{j, k}\right|^{2}\right)-2 \sum_{j, k} w_{j, k}\left|u_{j, k}\right|\left|v_{j, k}\right| \cos \left(\xi_{j, k}-\chi_{j}\right),
$$

with $\xi_{j, k}=\operatorname{angle}\left(u_{j, k} v_{j, k}^{\star}\right)$ the phase of the cross-product $u_{j, k} v_{j, k}^{\star}$. Introducing $\eta_{j, k}=w_{j, k}\left|u_{j, k}\right|\left|v_{j, k}\right|$, the original Problem (24) becomes equivalent to:

$$
\max _{\chi \in(-\pi,+\pi]^{N}}\left\{g(\chi)=\sum_{j, k} \eta_{j, k} \cos \left(\xi_{j, k}-\chi_{j}\right)\right\} .
$$

Expanding the cosine, the criterion to maximize can be put in the form:

$$
g(\chi)=\sum_{j, k} \eta_{j, k} \cos \left(\xi_{j, k}-\chi_{j}\right)=\sum_{j}\{\cos \chi_{j} \underbrace{\sum_{k} \eta_{j, k} \cos \xi_{j, k}}_{x_{j}}+\sin \chi_{j} \underbrace{\sum_{k} \eta_{j, k} \sin \xi_{j, k}}_{y_{j}}\},
$$


which shows that maximizing $g(\chi)$ in $\chi$ is separable in independent problems (one for each $j$ ). Inside the braces of the right hand side of Eq. (26), each term is the scalar product between the vectors $\left(\cos \chi_{j}, \sin \chi_{j}\right)^{\mathrm{t}}$ and $\left(x_{j}, y_{j}\right)^{\mathrm{t}}$. The scalar product being maximized if the two vectors are aligned, the solution of Problems $(24)$ and (25) is given by:

$$
(\forall j) \quad \widehat{\chi}_{j}=\max _{\chi_{j} \in(-\pi,+\pi]} g(\chi)=\operatorname{angle}\left(x_{j}+\mathrm{i} y_{j}\right),
$$

where angle $(z)$ yields the phase of the complex $z$ in the semi-open range $(-\pi,+\pi]$. Replacing $\chi$ by the best estimate $\widehat{\chi}$ in $f(\chi)$ and $g(\chi)$ yields:

$$
\begin{aligned}
\min _{\chi \in(-\pi,+\pi]^{N}} f(\chi) & =f(\widehat{\chi})=\sum_{j, k} w_{j, k}\left(\left|u_{j, k}\right|^{2}+\left|v_{j, k}\right|^{2}\right)-2 g(\widehat{\chi}), \\
\max _{\chi \in(-\pi,+\pi]^{N}} g(\chi) & =g(\widehat{\chi})=\sum_{j} \sqrt{x_{j}^{2}+y_{j}^{2}} \\
& =\sum_{j} \sqrt{\sum_{k, k^{\prime}} \eta_{j, k} \eta_{j, k^{\prime}} \cos \left(\xi_{j, k}-\xi_{j, k^{\prime}}\right)}
\end{aligned}
$$

where the last expression is obtained by expanding the squared sums and applying simple trigonometric identities.

\section{REFERENCES}

[1] Soulez, F., Thiebaut, É., Tallon, M., Tallon-Bosc, I., and Garcia, P., "Optimal a posteriori fringe tracking in optical interferometry," in [SPIE Astronomical Telescopes and Instrumentation], 9146, 91462Y, International Society for Optics and Photonics (Aug. 2014).

[2] Petrov, R. G., Malbet, F., Weigelt, G., Antonelli, P., Beckmann, U., Bresson, Y., Chelli, A., Dugué, M., Duvert, G., Gennari, S., Glück, L., Kern, P., Lagarde, S., Le Coarer, E., Lisi, F., Millour, F., Perraut, K., Puget, P., Rantakyrö, F., Robbe-Dubois, S., Roussel, A., Salinari, P., Tatulli, E., Zins, G., Accardo, M., Acke, B., Agabi, K., Altariba, E., Arezki, B., Aristidi, E., Baffa, C., Behrend, J., Blöcker, T., Bonhomme, S., Busoni, S., Cassaing, F., Clausse, J.-M., Colin, J., Connot, C., Delboulbé, A., Domiciano de Souza, A., Driebe, T., Feautrier, P., Ferruzzi, D., Forveille, T., Fossat, E., Foy, R., Fraix-Burnet, D., Gallardo, A., Giani, E., Gil, C., Glentzlin, A., Heiden, M., Heininger, M., Hernandez Utrera, O., Hofmann, K.-H., Kamm, D., Kiekebusch, M., Kraus, S., Le Contel, D., Le Contel, J.-M., Lesourd, T., Lopez, B., Lopez, M., Magnard, Y., Marconi, A., Mars, G., Martinot-Lagarde, G., Mathias, P., Mège, P., Monin, J.-L., Mouillet, D., Mourard, D., Nussbaum, E., Ohnaka, K., Pacheco, J., Perrier, C., Rabbia, Y., Rebattu, S., Reynaud, F., Richichi, A., Robini, A., Sacchettini, M., Schertl, D., Schöller, M., Solscheid, W., Spang, A., Stee, P., Stefanini, P., Tallon, M., Tallon-Bosc, I., Tasso, D., Testi, L., Vakili, F., von der Lühe, O., Valtier, J.-C., Vannier, M., and Ventura, N., "AMBER, the near-infrared spectro-interferometric three-telescope VLTI instrument," Astron. Astrophys. 464, 1-12 (Mar. 2007).

[3] Tatulli, E., Millour, F., Chelli, A., Duvert, G., Acke, B., Hernandez Utrera, O., Hofmann, K.-H., Kraus, S., Malbet, F., Mège, P., Petrov, R. G., Vannier, M., Zins, G., Antonelli, P., Beckmann, U., Bresson, Y., Dugué, M., Gennari, S., Glück, L., Kern, P., Lagarde, S., Le Coarer, E., Lisi, F., Perraut, K., Puget, P., Rantakyrö, F., Robbe-Dubois, S., Roussel, A., Weigelt, G., Accardo, M., Agabi, K., Altariba, E., Arezki, B., Aristidi, E., Baffa, C., Behrend, J., Blöcker, T., Bonhomme, S., Busoni, S., Cassaing, F., Clausse, J.-M., Colin, J., Connot, C., Delboulbé, A., Domiciano de Souza, A., Driebe, T., Feautrier, P., Ferruzzi, D., Forveille, T., Fossat, E., Foy, R., Fraix-Burnet, D., Gallardo, A., Giani, E., Gil, C., Glentzlin, A., Heiden, M., Heininger, M., Kamm, D., Kiekebusch, M., Le Contel, D., Le Contel, J.-M., Lesourd, T., Lopez, B., Lopez, M., Magnard, Y., Marconi, A., Mars, G., Martinot-Lagarde, G., Mathias, P., Monin, J.L., Mouillet, D., Mourard, D., Nussbaum, E., Ohnaka, K., Pacheco, J., Perrier, C., Rabbia, Y., Rebattu, S., Reynaud, F., Richichi, A., Robini, A., Sacchettini, M., Schertl, D., Schöller, M., Solscheid, W., Spang, A., Stee, P., Stefanini, P., Tallon, M., Tallon-Bosc, I., Tasso, D., Testi, L., Vakili, F., von der Lühe, O., Valtier, J.-C., and Ventura, N., "Interferometric data reduction with AMBER/VLTI. principle, estimators, and illustration," Astron. Astrophys. 464, 29-42 (2007).

[4] Gordon, J. A. and Buscher, D. F., "Detection noise bias and variance in the power spectrum and bispectrum in optical interferometry," Astron. Astrophys. 541, A46 (May 2012).

[5] Goodman, J. W., [Statistical optics] (1985).

[6] Chelli, A., Hernandez Utrera, O., and Duvert, G., "Optimised data reduction for the AMBER/VLTI instrument," A $6 A$ 502, 705-709 (Aug 2009).

[7] Petrov, R. G., [Diffraction-Limited Imaging with Very Large Telescopes], vol. 274 of NATO ASI Series, ch. Differential Interferometry, 249-272. 